\begin{tabular}{|c|l|}
\hline Title & Influence of shear flow on the linear response of a nematic liquid crystal to external electric fields \\
\hline Author(s) & Orihara, Hiroshi; Y ang, Fan; Takigami, Y uta; Takikawa, Y oshinori; Na, Y ang Ho \\
\hline Citation & $\begin{array}{l}\text { Physical Review E, 86(4), O41701 } \\
\text { https://doi.org/40.1103/PhysRevE.86.041701 }\end{array}$ \\
\hline Issue Date & 2012-10 \\
\hline Doc URL & http://hdl.handle.net/2115/50401 \\
\hline Rights & ○ 2012 A merican Physical Society \\
\hline Type & article \\
\hline File Information & PRE86-4_041701.pdf \\
\hline
\end{tabular}

Instructions for use 


\title{
Influence of shear flow on the linear response of a nematic liquid crystal to external electric fields
}

\author{
Hiroshi Orihara, ${ }^{*}$ Fan Yang, Yuta Takigami, and Yoshinori Takikawa \\ Division of Applied Physics, Faculty of Engineering, Hokkaido University, Sapporo, Hokkaido 060-8628, Japan \\ Yang $\mathrm{Ho} \mathrm{Na}$ \\ Department of Advanced Materials, College of Life Science and Nano Technology, Hannam University, Jeonmin-dong 461-6, \\ Yuseong-gu, Daejeon 305-811, South Korea \\ (Received 30 June 2012; published 4 October 2012)
}

\begin{abstract}
We have investigated the linear response of shear stress to ac electric fields under shear flow in a nematic liquid crystal. The experimental results were compared with the theoretical results derived from the Ericksen-Leslie theory. Although close agreement was obtained at low shear rates, discrepancies were observed at high shear rates. By introducing a two-mode coupling model the experimental results were well reproduced for the entire range of shear rates, and nonconservative forces were found to play an important role in determining the fluctuation dynamics, which is a characteristic of nonequilibrium steady states.
\end{abstract}

DOI: 10.1103/PhysRevE.86.041701

PACS number(s): 42.70.Df, 47.57.Lj, 05.70.Ln, 05.40.-a

\section{INTRODUCTION}

The rheological properties of nematic liquid crystals (NLC's) have been extensively studied from both fundamental and practical points of view. The shear viscosity can be easily controlled by applying external fields such as electric and magnetic fields, which change the orientation of the director (the average molecular direction) with respect to the flow velocity and its gradient [1-6]. For example, some nematic liquid crystals with positive dielectric anisotropy (where the dielectric constant parallel to the director is larger than that perpendicular to it) show a substantial increase in apparent viscosity upon application of an external electric field owing to the alignment of the director along the field. This phenomenon, known as the electrorheological effect, is important for the application of liquid crystals in electromechanical devices. Although the applied electric fields are usually sufficiently strong to induce a notable change in the director, in this paper we apply a weak sinusoidal electric field as a perturbation to examine the fluctuations in orientation through the change of shear stress under steady shear flow.

Thus far, the response of shear stress to weak sinusoidal electric fields has been investigated in an immiscible polymer blend, in which one polymer is dispersed as droplets in the other [7]. The electric field modifies the shape of the droplets, resulting in changes in shear stress. In the dependence of the shear stress on the frequency, one characteristic relaxation was observed, which was not of the Debye type. The real part of the response function takes negative values under a steady shear flow at high shear rates, which is one of the remarkable features characteristic to nonequilibrium steady systems (NESS's). The negative real part was clarified to originate from normal modes with complex eigenvalues derived from the Maffettone-Minale model $[8,9]$. These modes appearing under shear flow were also observed in superimposed storage modulus measurements performed by Chen et al. [10]. The basic idea of this technique is to analyze the steady-flow behavior by applying a linear perturbation at various frequencies [11-14]. Usually the

*orihara@eng.hokudai.ac.jp oscillatory deformations can be superimposed either parallel or perpendicular to the main flow direction. In this manner, detailed information about the spectral content under the steady flow is obtained for soft matters such as polymers, colloids, liquid crystals, and surfactants. Our method, which uses ac electric fields as a perturbation, may be equivalent to the shear-superimposed one if the microstructure is changed by applying an electric field.

Other theoretical and experimental studies have also been conducted on fluctuations in NESS under shear flow by using systems other than immiscible blends [15-24]. For example, Sakaue et al. [16] performed a theoretical investigation of a model system consisting of two beads connected by a harmonic spring under shear flow, showing that nonconservative forces caused by the shear flow violate the fluctuation dissipation theory (FDT) and deriving an alternative relation valid for NESS's. Furthermore, Ziehl et al. [17] conducted experiments on the Brownian motion under shear flow of a particle held by optical tweezers and showed the presence of strong time asymmetry in the cross correlation of particle fluctuations perpendicular and parallel to streamlines. These examples clearly demonstrate that shear flow can easily create NESS, in which fluctuations are expected to exhibit peculiar behaviors different from that in equilibrium states.

In the following section we show the obtained response of shear stress to ac electric fields under steady shear flow. In Sec. III, the shear stress is derived using the EricksenLeslie theory [25] under simple assumptions, and the result is compared with measured results. Furthermore, the observed discrepancy is discussed in terms of a two-mode coupling model with nonconservative forces. The last section is devoted to a summary.

\section{EXPERIMENTAL AND RESULTS}

A liquid crystal [4- $n$-pentyl-4' -cyanobiphenyl (5CB), Wako Pure Chemical Industries] was used without further purification. Measurements were carried out with a parallel-plate rotational rheometer (Physica MCR300, Anton Paar) at $25^{\circ} \mathrm{C}$ [7]. The diameters of the rotating plate and the gap between 


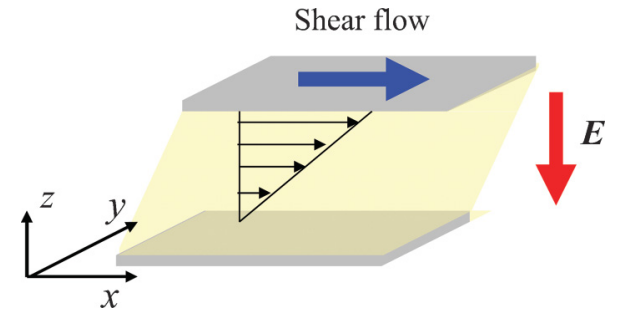

FIG. 1. (Color online) Simple shear flow is applied along the $x$ axis with a velocity gradient parallel to the $z$ axis. A weak sinusoidal electric field is applied along the $z$ axis as a perturbation.

two parallel plates were 35 and $0.2 \mathrm{~mm}$, respectively. Since we used a parallel-plate rotational viscometer and the shear rate depends on the position, the shear rate was defined at the periphery of the upper plate. The shear stress at the edge of the top plate was calculated from the torque by assuming that the fluid was Newtonian. Figure 1 shows the relation between the flow direction, the velocity gradient, and the electric field, which was applied to the sample by using a synthesizer (Model 1940, NF Electric Instruments) and a high-voltage amplifier (4005, NF).

Fluctuations in the director under steady shear flow were investigated by the application of an ac electric field $E(t)=$ $E_{0} \cos \omega t$ with an amplitude $E_{0}$ and an angular frequency $\omega$, in which case the shear stress may be written as

$$
\sigma(t)=\sigma_{0}+\sigma_{2,0}(\omega)+\operatorname{Re}\left[\sigma_{2,2}(\omega) e^{i 2 \omega t}\right],
$$

where $\sigma_{0}$ is the shear stress in the absence of an external electric field. Note that the stress is independent of the polarity of the electric field as the nematic liquid crystal is nonpolar and, therefore, the $2 \omega$ response appears and $\sigma_{2,0}(\omega)$ and $\sigma_{2,2}(\omega)$ should be proportional to the square of $E_{0}$ for small values of $E_{0}$. The $2 \omega$ component of the shear stress was measured with a vector signal analyzer (HP89410A, Hewlett-Packard).

Figure 2 shows the dependence of $\left|\sigma_{2,2}(\omega)\right|$ on the square of $E_{0}$ at $\omega=0.1 \times 2 \pi \mathrm{rad} \mathrm{s}^{-1}$ and $\dot{\gamma}=10 \mathrm{~s}^{-1}$. The linearity was confirmed to hold at small values of $E_{0}$. All measurements were performed at $E_{0}=14.1 \mathrm{~V} / \mathrm{mm}$. Typical dispersions of $\sigma_{2,2}(\omega)=\sigma_{2,2}^{\prime}(\omega)-i \sigma_{2,2}^{\prime \prime}(\omega)$ as a function of $2 \omega$ are shown in Fig. 3 at several shear rates. Although at low shear rates the frequency dispersion resembles a Debye-type dispersion,

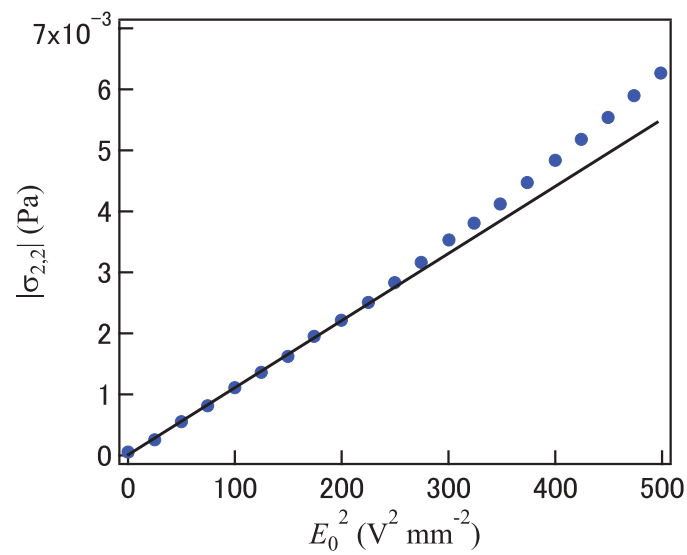

FIG. 2. (Color online) Dependence of $\left|\sigma_{2,2}(\omega)\right|$ on $E_{0}^{2}$. A linear relation is obtained at low electric fields. as the shear rate is increased the real part takes negative values for a certain frequency range, and the peak of the imaginary part becomes narrow. These characteristics at high shear rates are discussed in the following section. Remarkably, the peak position of the imaginary part shifts to higher frequencies as the shear rate is increased. As shown in Fig. 4, the peak frequency $2 \omega_{\text {peak }}$ is proportional to the shear rate at low shear rates.

\section{DISCUSSION}

In order to explain the frequency dispersion observed in a steady shear flow, first we derive $\sigma_{2,2}(\omega)$ using the Ericksen-Leslie theory [25]. For an incompressible nematic liquid crystal, the director field $\mathbf{n}(\mathbf{r}, t)$ and the velocity field $\mathbf{v}(\mathbf{r}, t)$ are described by the Ericksen-Leslie theory as follows:

$$
\begin{gathered}
\rho \frac{d}{d t} v_{\alpha}=\nabla_{\beta}\left(\sigma_{\beta \alpha}^{(e)}+\sigma_{\beta \alpha}^{(\mathrm{visc})}\right), \\
\mathbf{n} \times \mathbf{h}=\gamma_{1} \mathbf{n} \times \mathbf{N}+\gamma_{2} \mathbf{n} \times A \mathbf{n}, \\
\nabla_{\alpha} v_{\alpha}=0,
\end{gathered}
$$

with

$$
\begin{gathered}
\sigma_{\beta \alpha}^{(\mathrm{visc})}=\alpha_{4} A_{\alpha \beta}+\alpha_{1} n_{\alpha} n_{\beta} n_{\mu} n_{\rho} A_{\mu \rho}+\alpha_{5} n_{\alpha} n_{\mu} A_{\mu \beta} \\
+\alpha_{6} n_{\beta} n_{\mu} A_{\mu \alpha}+\alpha_{2} n_{\alpha} N_{\beta}+\alpha_{3} n_{\beta} N_{\alpha} \\
\sigma_{\beta \alpha}^{(e)}=-\frac{\partial f}{\partial\left(\nabla_{\beta} n_{\gamma}\right)} \nabla_{\alpha} n_{\gamma}-p \delta_{\alpha \beta} \\
h_{\alpha}=\nabla_{\beta}\left(\frac{\partial f}{\partial\left(\nabla_{\beta} n_{\alpha}\right)}\right)-\frac{\partial f}{\partial n_{\alpha}} \\
N_{\alpha}=\frac{d n_{\alpha}}{d t}-W_{\alpha \beta} n_{\beta}
\end{gathered}
$$

where the summation convention is used. Here, $d / d t$ denotes the material derivative, $\rho$ the density, $\sigma_{\beta \alpha}^{(e)}$ the Ericksen stress, $\sigma_{\beta \alpha}^{(\text {visc) }}$ the viscous stress, $f$ the free energy density, $p$ the pressure, $h_{\alpha}$ the molecular field, $N_{\alpha}$ the rate of change of the director with respect to the background fluid, $A_{\alpha \beta}=$ $1 / 2\left(\nabla_{\beta} v_{\alpha}+\nabla_{\alpha} v_{\beta}\right)$ the symmetric part of the velocity gradient, $W_{\alpha \beta}=1 / 2\left(\nabla_{\beta} v_{\alpha}-\nabla_{\alpha} v_{\beta}\right)$ the antisymmetric part of the velocity gradient, and $\alpha_{i}(i=1, \ldots, 6), \gamma_{1}=\alpha_{3}-\alpha_{2}, \gamma_{2}=\alpha_{6}-$ $\alpha_{5}$ the Leslie coefficients. For 5CB these coefficients have been determined [26]: $\alpha_{1}=-0.00767 \mathrm{~Pa} \mathrm{~s}, \alpha_{2}=-0.08171 \mathrm{~Pa} \mathrm{~s}$, $\alpha_{3}=-0.00433 \mathrm{~Pa} \mathrm{~s}, \alpha_{4}=0.06642 \mathrm{~Pa} \mathrm{~s}, \alpha_{5}=0.06725 \mathrm{~Pa} \mathrm{~s}$, $\alpha_{6}=-0.01879 \mathrm{~Pa} \mathrm{~s}$ at $25^{\circ} \mathrm{C}$, which is used in numerical calculations later.

Here we consider a simple case where the velocity gradient or the shear rate is constant and independent of space and time, and the director field is independent of space even when an external electric field is applied. In reality, the boundary conditions may give rise to spatially inhomogeneous velocity gradient and director fields. Specifically, in our experiments, the surfaces of the plates of the rheometer were not treated specially for director alignment. At present we do not have enough information on the director orientation. Polarizing microscopic observations were made by using 

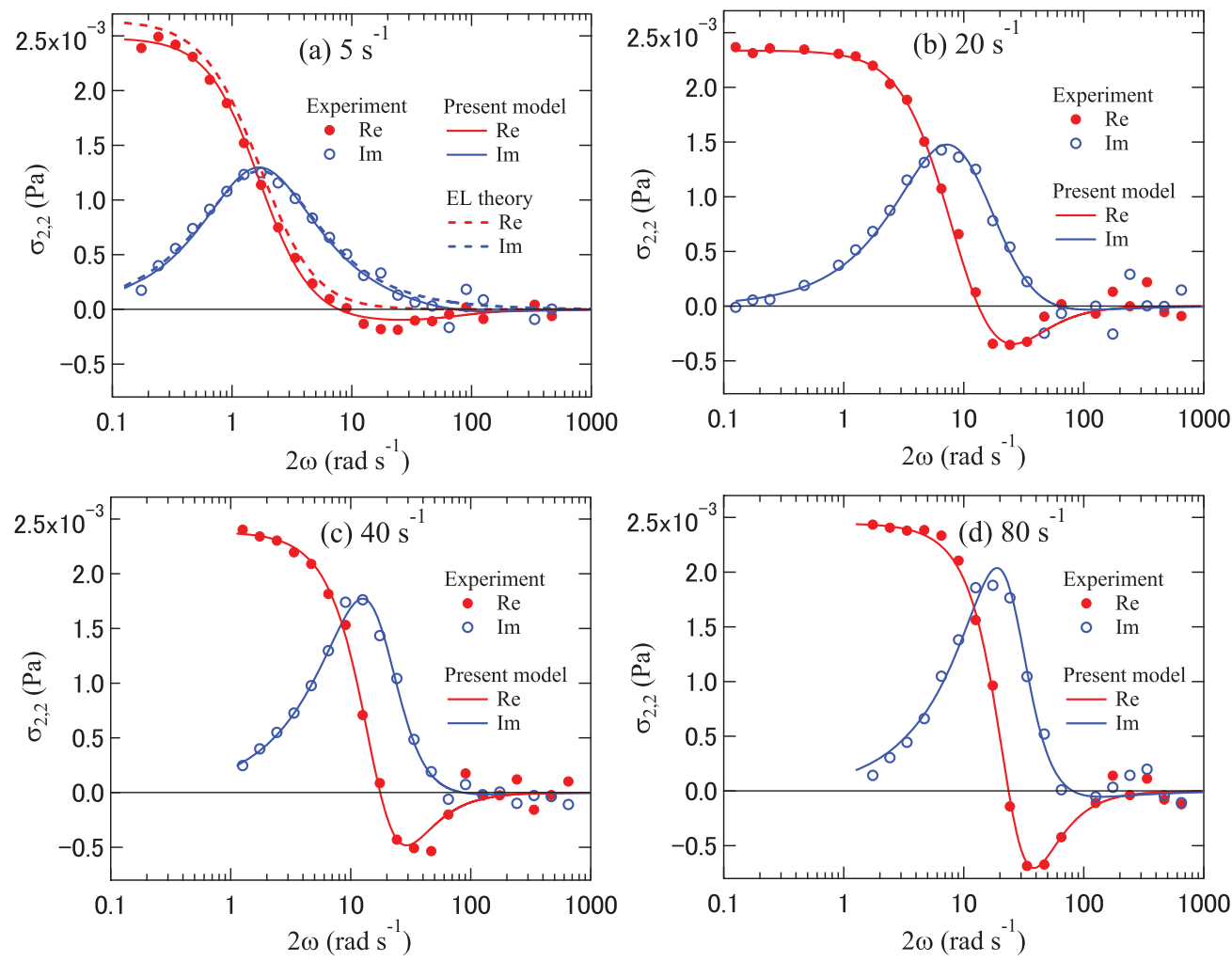

FIG. 3. (Color online) Frequency dispersions of $\sigma_{2,2}(\omega)$ as a function of $2 \omega$ at several shear rates for $E_{0}=14.1 \mathrm{~V} / \mathrm{mm}$. The dashed line in (a) is the result calculated using the Ericksen-Leslie theory, and the solid lines in all graphs are the best fits obtained with the two-mode coupling model.

another shearing device with optical windows. Under shear flow, director alignment was clearly observed, though there were some defects such as disclinations. It may be difficult to take into account these defects in the present analysis. Under the above assumptions, we can take $\mathbf{v}=(\dot{\gamma} z, 0,0)$ with a shear rate $\dot{\gamma}$ and $\mathbf{n}(t)=(\cos \theta(t), 0, \sin \theta(t))$ in the coordinate system shown in Fig. 1, which automatically satisfy Eqs. (2) and (4). For the spatially homogeneous case, there is no contribution from the Frank elastic energy, and only the electric energy

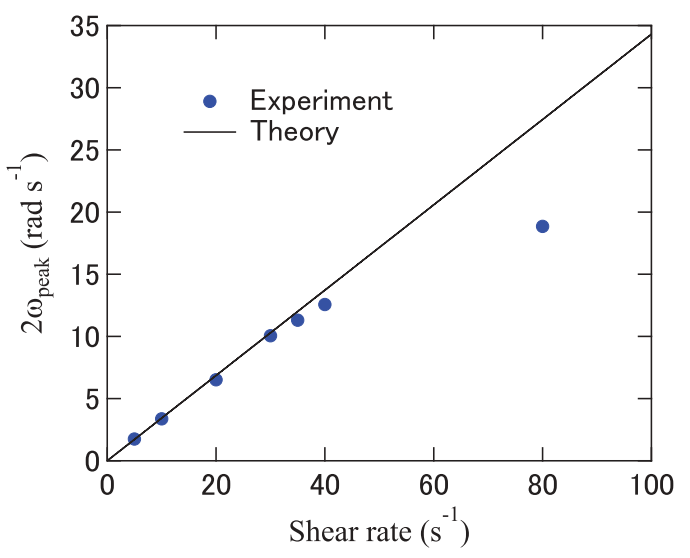

FIG. 4. (Color online) Dependence of $2 \omega_{\text {peak }}$ on the shear rate, where $2 \omega_{\text {peak }}$ is the peak angular frequency of the imaginary part in Fig. 3. A solid line is obtained from the Ericksen-Leslie theory. contributes to the free energy density:

$$
f=-\frac{1}{2} \varepsilon_{0} \varepsilon_{\perp} E^{2}-\frac{1}{2} \varepsilon_{0} \Delta \varepsilon(\mathbf{n} \cdot \mathbf{E})^{2},
$$

where $\varepsilon_{0}$ is the dielectric constant of vacuum and $\Delta \varepsilon(=11.5$ for 5CB [27]) the dielectric anisotropy defined as $\varepsilon_{\|}-\varepsilon_{\perp}$ with $\varepsilon_{\|}$and $\varepsilon_{\perp}$ being the dielectric constants parallel and perpendicular to the director, respectively. In this case, by using Eqs. (7) and (8), Eq. (3) is reduced to

$$
\gamma_{1} \frac{\partial \theta}{\partial t}=-\frac{1}{2} \dot{\gamma}\left(\gamma_{1}+\gamma_{2} \cos 2 \theta\right)+\frac{1}{2} \varepsilon_{0} \Delta \varepsilon E^{2} \sin 2 \theta,
$$

where the electric field $E$ is assumed to be applied in the $z$ direction. In the absence of an external electric field, the flow orientation angle $\theta_{0}$ in the steady state is given:

$$
\cos 2 \theta_{0}=-\frac{\gamma_{1}}{\gamma_{2}}
$$

For $5 \mathrm{CB}, \theta_{0}=13.0^{\circ}$ at $25^{\circ} \mathrm{C}$. Substituting $E=E_{0} \cos \omega t$ and $\theta=\theta_{0}+\Delta \theta$ into Eq. (10), we obtain an expression for $\Delta \theta$ up to the first order with respect to $E_{0}^{2}$ :

$$
\Delta \theta=\Delta \theta_{0}+\operatorname{Re}\left[\Delta \theta_{2} e^{i 2 \omega t}\right],
$$

with

$$
\begin{gathered}
\Delta \theta_{0}=-\frac{\varepsilon_{0} \Delta \varepsilon E_{0}^{2}}{4 \gamma_{2} \dot{\gamma}}, \\
\Delta \theta_{2}=\frac{\Delta \theta_{0}}{1+i 2 \omega \tau(\dot{\gamma})},
\end{gathered}
$$


where the relaxation time $\tau(\dot{\gamma})$ is given as

$$
\tau(\dot{\gamma})=-\frac{\gamma_{1}}{\gamma_{2} \dot{\gamma} \sin 2 \theta_{0}} .
$$

Thus, $\sigma_{0}, \sigma_{2,0}$, and $\sigma_{2,2}$ in Eq. (1) are calculated from $\sigma_{z x}^{(\text {visc })}$ in Eq. (5):

$$
\begin{gathered}
\sigma_{0}=\left[\alpha_{1} \sin ^{2} \theta_{0} \cos ^{2} \theta_{0}+\frac{1}{2}\left\{\alpha_{4}+\left(\alpha_{5}-\alpha_{2}\right) \sin ^{2} \theta_{0}\right.\right. \\
\left.\left.+\left(\alpha_{3}+\alpha_{6}\right) \cos ^{2} \theta_{0}\right\}\right] \dot{\gamma}, \\
\sigma_{0,2}=-\frac{\varepsilon_{0} \Delta \varepsilon \sin 2 \theta_{0}\left(\alpha_{1} \cos 2 \theta_{0}-\alpha_{2}-\alpha_{3}\right) E_{0}^{2}}{4 \gamma_{2}}, \\
\sigma_{2,2}(\omega)=\frac{\sigma_{0,2}}{1+i 2 \omega \tau(\dot{\gamma})},
\end{gathered}
$$

where we have used Parodi's relation: $\alpha_{6}-\alpha_{5}=\alpha_{2}+\alpha_{3}$.

Equation (18) is obviously of the Debye type. However, it can approximate the frequency dispersion observed at low shear rates. Theoretical curves calculated using Eq. (18) are plotted with dashed lines in Fig. 3(a). Here, we have used the following equation to obtain the apparent shear stress for the parallel-plate rheometer corresponding to the experimental result [28]:

$$
\sigma_{2,2}^{(R)}(\omega)=\frac{4}{\dot{\gamma}_{R}^{3}} \int_{0}^{\dot{\gamma}_{R}} \sigma_{2,2}(\omega, \dot{\gamma}) \dot{\gamma}^{2} d \dot{\gamma},
$$

where $\dot{\gamma}_{R}$ is the shear rate at the edge of the rotating disk. Substitution of Eq. (18) into the above equation yields

$$
\sigma_{2,2}^{(R)}(\omega)=\sigma_{2,2}(0) f\left(2 \omega \tau\left(\dot{\gamma}_{R}\right)\right)
$$

with

$$
f(x) \equiv \frac{4}{3}-2 i x-4 x^{2}+4 i x^{3} \log \left(1+\frac{1}{i x}\right) .
$$

The agreement between theory and experiment is close at low shear rates, indicating that our assumptions such as the constant velocity gradient are valid, and that the spatially homogeneous change in director $\Delta \theta$ should be the fundamental mode excited by the applied ac electric field. In addition, from Eqs. (15) and (20) it is readily seen that the peak frequency $2 \omega_{\text {peak }}$ of the imaginary part is proportional to the shear rate, and from Eq. (21) we numerically obtain $2 \omega_{\text {peak }}=0.74187 / \tau\left(\dot{\gamma}_{R}\right)$. At low shear rates, the experimental data are in good agreement with the theoretical calculations, as shown in Fig. 4. On the other hand, the discrepancy between theory and experiment at high shear rates means the breakdown of our assumptions. Although the experimental results at high shear rates could be also explained with the Ericksen-Leslie theory, it is difficult to take into account the presence of defects, the spatial dependence of director, and so on. Therefore, we use a simple phenomenological model to analyze the experimental results.

We have previously observed a similar frequency dispersion with a negative real part in an immiscible polymer blend, where one polymer is dispersed as droplets in the other [7]. The negative real part was clarified to be due to normal modes with complex eigenvalues, which originate from the shear flow. More than one mode (degree of freedom) is necessary for complex eigenvalues to appear. These facts suggest that the following model applies in the present study. We assume another mode $\Delta \theta^{\prime}$ in addition to $\Delta \theta$, and although we do not specify $\Delta \theta^{\prime}$ here, the tilt angle of the director with respect to the $y$ direction is the most plausible choice. However, it should be noted that in the theoretical considerations, $\Delta \theta$ is independent of the fluctuation in the tilt of the director with respect to the $y$ direction, i.e., there is no correlation between $\Delta \theta$ and $\Delta \theta^{\prime}$. This is the result of the assumption that the director alignment is spatially homogeneous and the average orientation of the director has no $y$ component. In our experiments, however, the director orientation may have been disturbed since no surface treatment was performed to align liquid crystalline molecules, resulting in deviation from the ideal flow alignment. From the Ericksen-Leslie theory, a nonzero $y$ component of the director in the steady state can be shown to define the correlation between $\Delta \theta$ and $\Delta \theta^{\prime}$ [29]. For these fluctuations, we assume the following set of equations of motion:

$$
\begin{aligned}
\gamma_{1} \frac{d \Delta \theta}{d t} & =-a_{11} \Delta \theta-a_{12} \Delta \theta^{\prime}+\operatorname{Re}\left[K_{1} e^{i 2 \omega t}\right], \\
\gamma^{\prime} \frac{d \Delta \theta^{\prime}}{d t} & =-a_{21} \Delta \theta-a_{22} \Delta \theta^{\prime}+\operatorname{Re}\left[K_{2} e^{i 2 \omega t}\right],
\end{aligned}
$$

where we have omitted the dc terms responsible for $\Delta \theta_{0}$ in Eq. (12). Here $\gamma^{\prime}$ is the viscosity coefficient for $\Delta \theta^{\prime}, a_{i j}$ denote coefficients dependent on the shear rate, and $K_{i}$ represent the electric force. It should be noted that $a_{12} \neq a_{21}$ in the present system since nonconservative forces may be involved, although $a_{12}=a_{21}$ for equilibrium systems, in which case $a_{i j}$ is derived from the free energy $F$ as $a_{12}=\partial^{2} F / \partial \Delta \theta \partial \Delta \theta^{\prime}=$ $a_{21}$. In addition, we assume that the shear stress $\sigma$ is obtained by a linear combination of $\Delta \theta$ and $\Delta \theta^{\prime}: \sigma=c_{1} \Delta \theta+c_{2} \Delta \theta^{\prime}$, where $c_{i}$ are coefficients depending on the shear rate. From these equations, we obtain

$$
\sigma_{2,2}(\omega)=\frac{i 2 \omega P_{1}+P_{2}}{(i 2 \omega)^{2}+i 2 \omega P_{3}+P_{4}},
$$

where $P_{i}$ are constants consisting of $a_{i j}, K_{i}$, etc. This can be rewritten as

$$
\sigma_{2,2}(\omega)=\frac{A_{1}}{1+i 2 \omega / \lambda_{1}}+\frac{A_{2}}{1+i 2 \omega / \lambda_{2}}
$$

where $\lambda_{i}$ and $A_{i}$ are given:

$$
\lambda_{1}, \lambda_{2}=\frac{a_{11} / \gamma_{1}+a_{22} / \gamma^{\prime} \pm \sqrt{\left(a_{11} / \gamma_{1}-a_{22} / \gamma^{\prime}\right)^{2}+4 a_{12} a_{21} /\left(\gamma_{1} \gamma^{\prime}\right)}}{2},
$$




$$
\begin{aligned}
& A_{1}=\frac{\lambda_{1} P_{1}-P_{2}}{\lambda_{1}\left(\lambda_{1}-\lambda_{2}\right)}, \\
& A_{2}=-\frac{\lambda_{2} P_{1}-P_{2}}{\lambda_{2}\left(\lambda_{1}-\lambda_{2}\right)} .
\end{aligned}
$$

Note that $\lambda_{1}$ and $\lambda_{2}$ are the eigenvalues of the following matrix:

$$
\left(\begin{array}{ll}
a_{11} / \gamma_{1} & a_{12} / \gamma_{1} \\
a_{21} / \gamma^{\prime} & a_{22} / \gamma^{\prime}
\end{array}\right),
$$

and $\exp \left(-\lambda_{1} t\right)$ and $\exp \left(-\lambda_{2} t\right)$ are the solutions to Eqs. (22) in the absence of external forces, that is, $K_{1}=K_{2}=0$. From Eq. (25), it is readily seen that the eigenvalues $\lambda_{1}$ and $\lambda_{2}$ are complex when the argument of the square root is negative, which requires $a_{12} a_{21}<0$.

Next, we fit the experimental data to Eq. (23). In strict terms, for the fit we should use $\sigma_{2,2}^{(R)}(\omega)$ as obtained from Eq. (19), which takes into account the shear rate distribution in the parallel plates. However, this is impossible as our model does not take into account the shear rate dependence of $a_{i j}$, etc. The fitting results are shown by solid lines in Fig. 3, revealing a close agreement for the entire range of shear rates, in which the two characteristic features, namely the negative real part and the peak narrowing, were well reproduced.
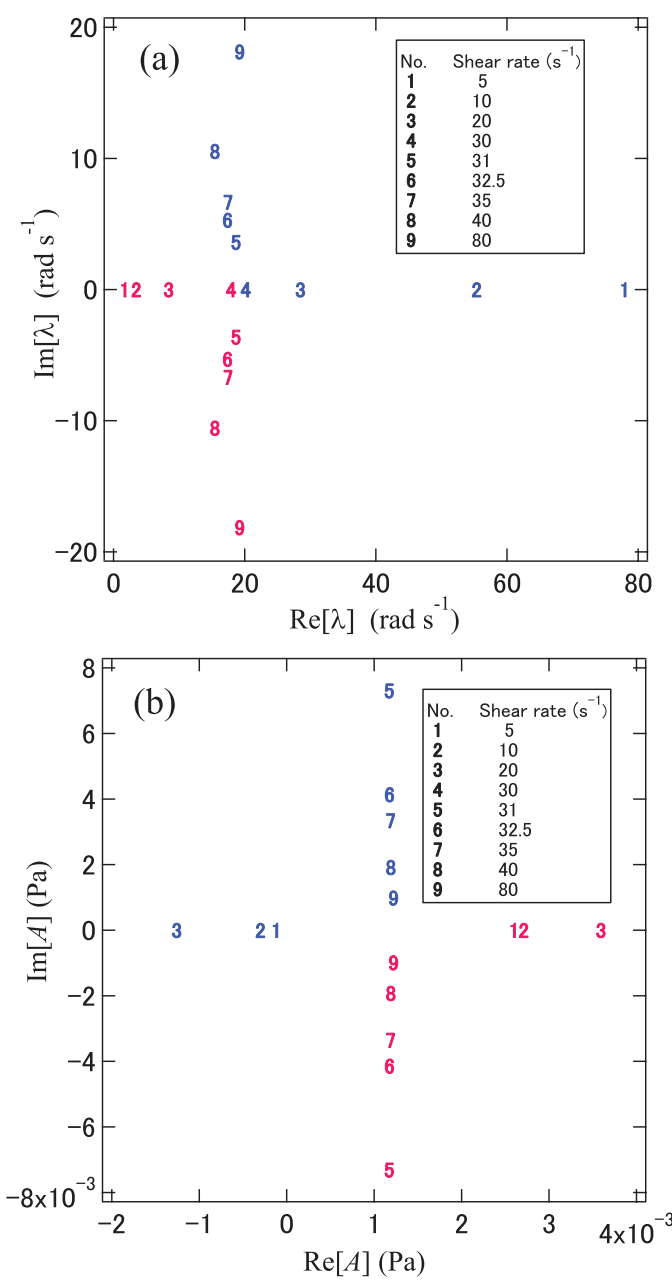

FIG. 5. (Color online) Shear rate dependencies of (a) the eigenvalues and (b) the strengths in the complex plane. Note that data points $\left(A_{1}=0.0256 \mathrm{~Pa}, A_{2}=-0.0233 \mathrm{~Pa}\right)$ at $30 \mathrm{~s}^{-1}$ are out of range.

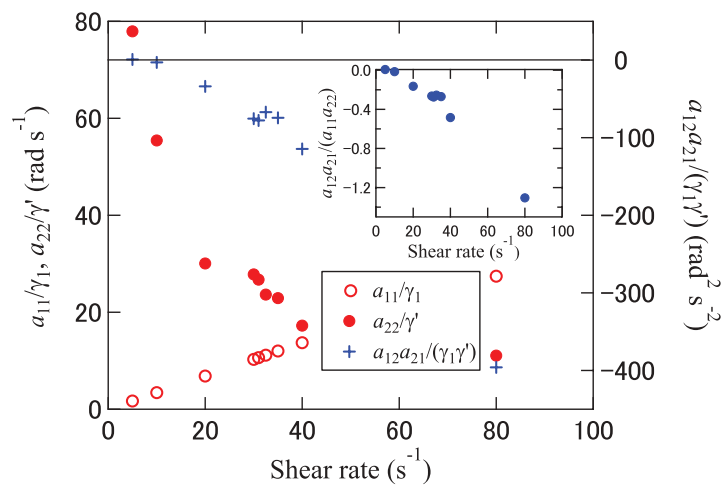

FIG. 6. (Color online) Shear rate dependencies of $a_{11} / \gamma_{1}, a_{22} / \gamma^{\prime}$, and $a_{12} a_{21} /\left(\gamma_{1} \gamma^{\prime}\right)$, where we assume $a_{11} / \gamma_{1}=0.74187 / \tau\left(\dot{\gamma}_{R}\right)$. The shear rate dependence of $a_{12} a_{21} /\left(a_{11} a_{22}\right)$ is also shown in the inset.

Furthermore, $A_{i}$ and $\lambda_{i}$ were calculated from $P_{i}$ obtained from the fits. Figure 5(a) shows the shear rate dependencies of the eigenvalues in the complex plane. At low shear rates, both eigenvalues are real, and the smaller eigenvalue may correspond to that of $\Delta \theta$. As the shear rate is increased, they approach each other and eventually degenerate at around $\dot{\gamma}=$ $30 \mathrm{~s}^{-1}$. Then, the eigenvalues depart from the real axis and become complex conjugates. This fact clearly indicates that nonconservative forces should exist at least at high shear rates since $a_{12} a_{21}<0$ and thus $a_{12} \neq a_{21}$. The corresponding $A_{1}$ and $A_{2}$ are shown in Fig. 5(b). At $5 \mathrm{~s}^{-1}$, the low frequency mode is notably stronger than the high frequency mode, and the latter takes negative values, which is responsible for the negative real part in the frequency dispersion. When the shear is increased, the low frequency mode increases in strength, whereas the high frequency mode decreases. It appears that both modes diverge on the real axis when the shear rate of the degenerate point is approached from below, and on the imaginary axis when it is approached from above. This behavior can be readily understood from Eq. (26). Figure 3 shows that the peak of the imaginary part becomes sharper as the shear rate is increased, particularly when the eigenvalues become complex. The peak width can be easily shown to become zero when the argument of $\lambda$ becomes $\pi / 2$.

Equation (25) gives

$$
\begin{aligned}
\lambda_{1}+\lambda_{2} & =a_{11} / \gamma_{1}+a_{22} / \gamma^{\prime}, \\
\lambda_{1} \lambda_{2} & =\left(a_{11} / \gamma_{1}\right)\left(a_{22} / \gamma^{\prime}\right)-\left(a_{12} / \gamma_{1}\right)\left(a_{21} / \gamma^{\prime}\right) .
\end{aligned}
$$

From Eq. (28a) we can obtain the relaxation rate of $\Delta \theta^{\prime}, a_{22} / \gamma^{\prime}$, if we assume that $a_{11} / \gamma_{1}$ is the inverse of $\tau(\dot{\gamma})$ in Eq. (15). In reality, we should assume $a_{11} / \gamma_{1}=0.74187 / \tau\left(\dot{\gamma}_{R}\right)$ instead, owing to the correction for the parallel-plate rheometer. Then, we can calculate $\left(a_{12} / \gamma_{1}\right)\left(a_{21} / \gamma^{\prime}\right)$ from Eq. (28b). The respective dependencies of $a_{11} / \gamma_{1}=0.74187 / \tau\left(\dot{\gamma}_{R}\right), a_{22} / \gamma^{\prime}$, and $a_{12} a_{21} /\left(\gamma_{1} \gamma^{\prime}\right)$ on the shear rate are shown in Fig. 6 . It is clear that the relaxation rate of $\Delta \theta^{\prime}$ decreases monotonically as the shear rate is increased, in contrast to that of $\Delta \theta$. Furthermore, $a_{12} a_{21} /\left(\gamma_{1} \gamma^{\prime}\right)$, which represents the coupling strength between the two modes, takes negative values except at $5 \mathrm{~s}^{-1}$ (the value is almost zero) and its absolute value increases with the shear rate. In the inset of Fig. 6 , a relative coupling coefficient defined as $\left(a_{12} a_{21}\right) /\left(a_{11} a_{22}\right)$ is plotted as a function of the shear rate, which also decreases monotonically 
and takes large absolute values at high shear rates. The above results indicate that nonconservative forces should appear under shear flow and increase in magnitude together with the shear rate.

\section{SUMMARY}

We measured the response of shear stress to ac electric fields to investigate the fluctuations in orientation of a nematic liquid crystal 5CB under steady shear flow. At low shear rates, the frequency dispersion is of the Debye type, which is well explained by the Ericksen-Leslie theory. On the other hand, at high shear rates the frequency dispersion deviates from the Debye type as the real part of the response takes negative values and the peak of the imaginary part becomes narrow. These behaviors were well reproduced by a two-mode coupling model with nonconservative forces. Upon analyzing the data it was found that the eigenvalues of the modes become complex above a certain shear rate, clearly indicating the existence of nonconservative forces induced by shear flow.

In this study, we investigated the fluctuations in orientation on the basis of the response to external electric fields. In equilibrium systems, fluctuations and responses are related by the fluctuation-dissipation theory. In nonequilibrium steady states, however, this relation fails to hold and must be modified [16,30-34]. Therefore, in order to fully understand the nature of fluctuations in orientation under shear flow, the fluctuations should be observed directly, say, with the dynamic light scattering technique for nematic liquid crystals.

\section{ACKNOWLEDGMENTS}

We thank T. Ohta and T. Sakaue for useful discussions. This work was partially supported by Grant-in-Aid for Scientific Research Priority Area "Soft Matter Physics" Contract No. 463 and C Contract No. 22540416 from the Ministry of Education, Culture, Sports, Science and Technology of Japan.
[1] I. K. Yang and A. D. Shine, J. Rheol. 36, 1079 (1992).

[2] K. Negita, Chem. Phys. Lett. 246, 353 (1995).

[3] R. Kishi, T. Kitano, and H. Ichijo, Mol. Cryst. Liq. Cryst. Sci. Technol., Sect. A 280, 109 (1996).

[4] K. Negita, J. Chem. Phys. 105, 7837 (1996).

[5] Y. C. Chiang, A. M. Jamieson, S. Campbell, Y. H. Lin, N. Osidocky, L. C. Chien, M. Kawasumi, and V. Percec, Rheol. Acta 36, 505 (1997).

[6] Y. C. Chiang, A. M. Jamieson, M. Kawasumi, and V. Percec, Macromolecules 30, 1992 (1997).

[7] Y. H. Na, K. Aida, R. Sakai, T. Kakuchi, and H. Orihara, Phys. Rev. E 80, 061803 (2009).

[8] P. L. Maffettone and M. Minale, J. Non-Newtonian Fluid Mech. 78, 227 (1998).

[9] P. L. Maffettone and M. Minale, J. Non-Newtonian Fluid Mech. 84, 105 (1999).

[10] Q. Chen, W. Yu, and C. X. Zhou, J. Polym. Sci., Part B: Polym. Phys. 46, 431 (2008).

[11] J. Vermant, L. Walker, P. Moldenaers, and J. Mewis, J. NonNewton. Fluid Mech. 79, 173 (1998).

[12] J. Mewis, B. Kaffashi, J. Vermant, and R. J. Butera, Macromolecules 34, 1376 (2001).

[13] V. J. Anderson, J. R. A. Pearson, and J. D. Sherwood, J. Rheol. 50, 771 (2006).

[14] P. Ballesta, M. P. Lettinga, and S. Manneville, J. Rheol. 51, 1047 (2007).

[15] G. Taylor, Proc. R. Soc. London A 219, 186 (1953).

[16] T. Sakaue and T. Ohta, Phys. Rev. E 77, 050102 (2008).
[17] A. Ziehl, J. Bammert, L. Holzer, C. Wagner, and W. Zimmermann, Phys. Rev. Lett. 103, 230602 (2009).

[18] M. Fuchs and M. E. Cates, J. Rheol. 53, 957 (2009).

[19] T. Speck and U. Seifert, Phys. Rev. E 79, 040102 (2009).

[20] M. Thiebaud and T. Bickel, Phys. Rev. E 81, 031602 (2010).

[21] M. Kruger, F. Weysser, and T. Voigtmann, Phys. Rev. E 81, 061506 (2010).

[22] L. Holzer, J. Bammert, R. Rzehak, and W. Zimmermann, Phys. Rev. E 81, 041124 (2010).

[23] G. Szamel and M. Zhang, Europhys. Lett. 96, 50007 (2011).

[24] H. Orihara and Y. Takikawa, Phys. Rev. E 84, 061120 (2011).

[25] P. G. de Gennes and J. Prost, The Physics of Liquid Crystals (Clarendon, Oxford, United Kigndom, 1993).

[26] G. Ahlers, In Pattern Formation in Liquid Crystals, edited by L. Kramer and A. Buka (Springer, Berlin, 1996).

[27] P. G. Cummins, D. A. Dunmur, and D. A. Laidler, Mol. Cryst. Liq. Cryst. 30, 109 (1975).

[28] R. B. Bird, R. C. Armstrong, and O. Hassager, Dynamics of Polymeric Liquids (Wiley-Interscience, New York, 1987), Vol. 1.

[29] J. F. Fatriansyah and H. Orihara (unpublished).

[30] J. R. Gomez-Solano, A. Petrosyan, S. Ciliberto, R. Chetrite, and K. Gawedzki, Phys. Rev. Lett. 103, 040601 (2009).

[31] J. Mehl, V. Blickle, U. Seifert, and C. Bechinger, Phys. Rev. E 82, 032401 (2010).

[32] U. Seifert and T. Speck, Europhys. Lett. 89, 10007 (2010).

[33] H. D. Feng and J. Wang, J. Chem. Phys. 135, 234511 (2011).

[34] D. Chaudhuri and A. Chaudhuri, Phys. Rev. E 85, 021102 (2012). 